\title{
Automatic Estimation of Topic Flow from Slides for Supporting Presentation Slide Creation
}

\author{
Tomoko Kojiri* and Fumihiro Yamazoe*
}

(Received 15 June 2012 and accepted in revised form 17 January 2013)

\begin{abstract}
Researchers often present their ideas using presentation tools such as Microsoft Office PowerPoint. However, listeners sometimes cannot follow the story and cannot understand what the speakers intend, even if topics are appropriately selected and arranged. Such situations are caused by inappropriate representation of sentences that describe topics. The audience cannot re-organize topic structures based on the descriptions on the slides. Our research is aimed at developing a system that automatically detects differences in the speaker's (or author's) intention for topics and a topic structure that can be estimated from created slides. We focus on a mechanism for automatic estimation of the relationships between slides, especially sequential and inclusive relationships. Relationships between slides can be inferred by the change in focus in the topic structure, and the topic structure is estimated using lexical information on the slides. Therefore, we introduce a topic graph that represents the relationships among topics on slides, which is formed using lexical and layout information of slides. Relationships between created slides are detected by the change in their focused topics in the topic graph. Based on an experiment, we validated the detected relationships with a prototype system when slides mainly consist of typical topics of a presentation and do not contain compound nouns.
\end{abstract}

Keywords: presentation slide creation support, automatic estimation of topic flow, topic graph, gap between author's intention and slide structure

\section{Introduction}

In many situations, researchers present their ideas using presentation tools such as Microsoft Office PowerPoint. In a presentation, most of the content is simply organized into presentation slides and details are explained by the speaker (or author). Presentation files consist of a sequence of presentation slides (slides) in which descriptions of topics are organized sequentially. The author of the slides (author) creates the presentation story (story) based on the topics that he/she wants to explain. Then, he/she generates content, such as text, diagrams, or tables that explain the topics by considering the relationships among them. However, an audience sometimes cannot understand the story that the author intends by looking at the slides. In many cases, it is difficult for authors to detect the inappropriateness of their slides. Thus, being able to point out the differences between an author's intention and an audience's understanding is valuable.

There have been several studies that support au-

\footnotetext{
* Faculty of Engineering Science, Kansai University, Japan
}

thors for creating logical presentation slides ${ }^{(1,2)}$. For example, Maeda et al. ${ }^{(3)}$ constructed a collaborative learning environment for discussing slide story generation. They proposed a story amendment tool that assists an audience (students for learning story creation knowledge) in re-organizing the presented slides to modify them. Also, they developed a discussion support tool that shows modified slides visually to promote meaningful discussion. In this environment, whether students can effectively discuss story creation knowledge depends on their abilities. Therefore, students sometimes are not able to acquire slide creation knowledge. On the other hand, Kamewada and Nishimoto ${ }^{(4)}$ constructed a tool that extracts audience members' eye movements during a presentation. By being provided with the differences in slide presentation and listeners' eye directions, authors can see the inappropriateness of their stories. However, to modify slides using this system, authors need to present the slides in front of a live audience. Therefore, this system cannot be used when there is no audience.

Hanaue and Watanabe ${ }^{(5)}$ constructed a system that creates slides automatically. In this system, the author has to input topics and their relationships beforehand. Then, slides that satisfy the author's intention can be 
generated. However, the author needs to input a topic structure beforehand. Also, the author's skills for creating slides do not improve. Therefore, he/she needs to use the system every time that slides are created.

Our research is aimed at constructing a system that fosters slide creation indirectly by automatically pointing out conflicts between topic structures estimated by created slides and the inputted author's intention. By considering the reason for detected inappropriateness, authors can acquire skills for creating appropriate slides.

For listeners, especially for presentations for which the content is mostly described in the slides, relationships among slides can be inferred from the descriptions on the slides. For instance, if one slide (explaining slide) explains a topic that is already described on another slide (explained slide), these slides contain the same words and the explaining slide has several sentences that explain the common words. To model the audience's understanding of slides, our system automatically analyzes the descriptions on the slides and detects the relationships among topics. By comparing them with the author's intention for topics, conflicts between the author's intention and generated slides can be detected.

In Section 2, the overall framework of our system is described. To estimate the relationships between slides, the system must form a topic structure from them. A topic graph is introduced for representing topics and their relationships. In Section 3, the details of the topic graph are explained. In Section 4, a method for estimating relationships between slides based on the topic graph is discussed. Evaluation of detected relationships is discussed in Section 5. Finally, the paper is concluded in Section 6.

\section{Framework}

\subsection{Slide structure}

When creating slides, authors prepare many topics related to the presentation theme. Authors select topics from the topic structure to include on the slides, arrange them sequentially to generate a story that is easy to understand, and explain them. However, even if topics are selected and arranged appropriately, the content of the presentation is still not understood by the audience if the topics are described inappropriately.

There are two types of inappropriate descriptions that prevent the audience from understanding the con-

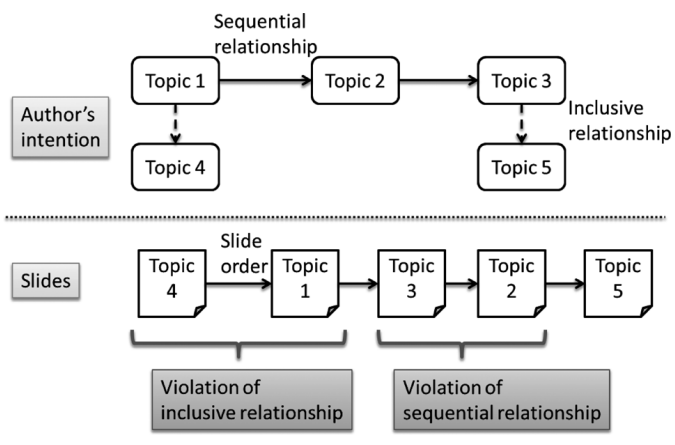

Figure 1. Example of Inappropriate Slide Order.

tent. One is the inappropriateness of explaining each topic and the other is the lack of indicating the relationships between related slides. As Tufte ${ }^{(6)}$ described, relationships among slides are difficult to represent when creating slides. If relationships between slides are expressed clearly, such as "approach (1)" and "approach (2)", it is easy to grasp the relationship. If slides contain similar expressions or words, the audience members can estimate the relationships. However, for example, if authors use different words for the same meaning on a different slide, listeners may find it difficult to notice that these slides are related.

The goal of our research is to analyze whether author intentions for slides and those grasped by the audience from the slides are the same. If the relationships are determined as different, our research also provides support for authors to modify the slide descriptions. Currently, the target author's intention is the relationship between topics on each slide, such as sequential and inclusive. A sequential relationship means that the topic transforms to another related topic. The former topic in the sequential relationship is often a requirement to understanding the latter topic, so the former topic should come before the latter in the slide sequence. On the other hand, an inclusive relationship indicates that one topic (included topic) contains the details of another slide (including topic). The included topic sometimes cannot be understood if the including topic is not explained. Therefore, the including topic should come before the included topic.

Figure 1 illustrates the inappropriate slide order for each relationship. Let us assume that the upper part of Figure 1 illustrates the author's intention for topics and the lower part corresponds to the created slides. Since 
topic 4 is before topic 1 , the order is inappropriate because topic 1 includes topic 4 in the author's intention. Also, the order of topics 3 and 2 is also inappropriate since it is opposite to the author's intention.

\subsection{Approach}

Figure 2 shows the overall framework of our system. The objective of our system is to estimate relationships between slides in order to detect the conflict between created slides and the author's intention. The system contains a mechanism of estimating relationships between slides and compares the estimated relationships and author's intention for the slides. The differences are given to the author to allowing him/her to modify the slides and acquire slide creation skills experientially.

The following are types of presentations that we are currently focusing on.

1. Most of the content of the topics is described on the slides; content can be understood by looking at slides and only supplemental explanations are given by the author.

2. Each slide mainly consists of text with an itemization layout; content can be understood without understanding tables or diagrams on slides. Moreover, sentences are organized using the itemization layout provided by the presentation tool. We know that tables and diagrams are also important slide elements. Our future work will deal with tables and diagrams.

3. Each topic is described by a few sentences; content is decomposed into minimum levels and those levels are described with a simple sentence or sentences. We do not focus on slides that explain one topic with many sentences.

In such slides, the relationships between slides are estimated from descriptions on the created slides. New words appear when a new topic is proposed, while an explaining topic slide contains words that are already used in explained topics. Currently, we regard each itemized sentence or sentences on slides as individual topics. Based on the appearance and relationships between words in the itemized sentence or sentences, relationships among topics are inferred and a topic graph is constructed. A topic graph represents the relationships between topics. Topics with sequential relationships are located as sibling nodes and topics of inclusive relationships are located as child nodes.

Relationships of slides can be understood through

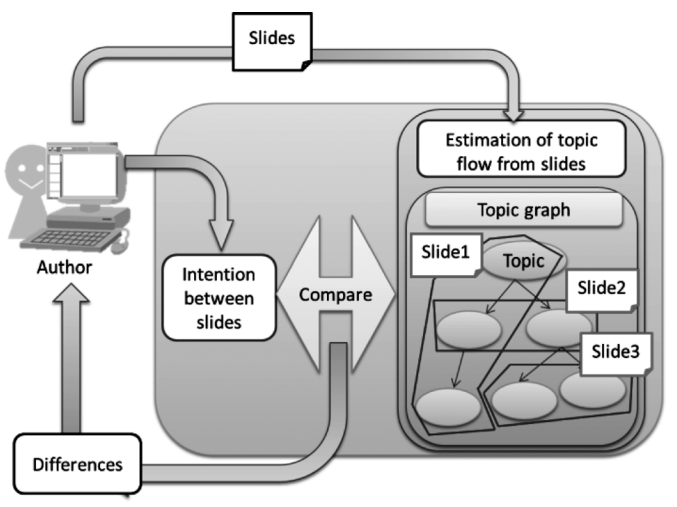

Figure 2. Framework of System.

focus points of all nodes on the slides in the topic graph. By comparing the position of the focus points for each slide, relationships between slides can be estimated.

Currently, we focus on the mechanism of estimating relationships between slides. The method of giving feedback to the author is not taken into consideration. In addition, acquisition of the author's intention is also for future work.

\section{Topic Graph}

A topic graph organizes topics and their relationships as understood from the created slides. Nodes correspond to each topic and links represent relationships: inclusive and sequential. The structure of a topic graph corresponds to the topic structure that the audience understands from the slides. Here, a topic indicates a sentence or a set of a few sentences that are organized as one unit to explain one item. On slides, each itemized sentence forms a topic, so nodes in the topic graph correspond to each itemized sentence. Even if itemized sentences consist of several sentences, they are regarded as one topic since they are intentionally gathered as one item. In addition, the topic structure can be understood by analyzing the relationships among itemized sentences.

Relationships among topics can be understood basically by their layout information. If the itemized level of one topic is lower than that of the other, the former topic may explain the latter topic. Therefore, the lower topic is a part of the upper topic. When topics are in the same itemized level, their relationships are understood 
due to their physical positions. Topics change from upper to lower, so that a sequential relationship is attached to these topics. Relationships are also defined between slides. A sequential relationship exists from topics on one slide to those on the next slide. In spite of attaching links to all pair of topics on both slides, one link is set between the nodes corresponding to their first itemized sentences, since they are representative topics of the slides. These relationships of topics correspond to what we can see in the outline mode of Microsoft Office PowerPoint.

Sometimes topics on one slide (explaining topic) explain the topics on a different slide (explained topic). In such a case, the topics should be re-organized to make the explaining node become a sub-graph of the explained node. The following are steps for re-organizing topics.

Step 1: Detect keywords that characterize each topic

Step 2: Detect nodes of the same topic based on the existence of common keywords

Step 3: Organize the topic graph to make nodes of the same topic have a relationship with each other

In Step 1, words that characterize the topics are extracted to detect the nodes of the same topic. Keywords are words that are often used in the topic but are not used in other topics. To detect such unique words, the term frequency-inverse document frequency $(t f-i d f)$ $\operatorname{method}^{(7)}$ is applied. The following equations are used to calculate the uniqueness of word $_{i}$ :

Uniqueness $_{i}=t f_{i} \cdot t d f_{i}$

$t f_{i}=\frac{\text { Number of } \text { word }_{i} \text { in sentence/s }}{\text { Number of all words in sentence/s }}$

$i d f_{i}=\log \frac{\text { Number of sentences in slides }}{\text { Number of sentences } \text { which include } \text { word }_{i}}$

The uniqueness of word $_{i}$ is calculated by multiplying its $t f$ and $i d f$ values. The $t f$ value is calculated as the ratio of the word $_{i}$ in the sentence or sentences of a topic. The idf value represents a distribution of words on a slide and is derived by the ratio of the sentences in the slide that include $\operatorname{word}_{i}$. If the uniqueness of a word is larger than the threshold, the word is regarded as a keyword.

In Step 2, nodes of the same topic are detected using their keywords. Since sentences consist of a limited number of words, nodes are regarded as similar top- ics if they contain the same keywords. Therefore, nodes that have more than one keyword in common are detected as the same topic.

In Step 3, the relationships between topics are modified if a topic on one slide explains a topic on another slide. The explaining topic may appear on the slide after the explained topic. In addition, the explaining topic may be located in the upper part in the topic graph because its detailed explanation should follow as its subgraph. The amount of explanation is acquired by the size of its sub-graph, namely all nodes that are acquired by following inclusive relationships. Therefore, if two nodes are regarded as the same topic in Step 2 and the latter node has a certain amount of explanation the subgraph of the latter node is moved as the sub-graph of the former node. The following is the equation for calculating the importance of node $e_{j}$, which indicates the amount of explanation of that node:

$$
\text { Importance }_{j}=\frac{(1 / 2)^{\text {level }_{j}}}{\sum_{k \in \text { all levelsin slides }} \text { number of topic }_{k} \times(1 / 2)^{k}}
$$

where level $_{j}$ represents the itemized level of node ${ }_{j}$ and topic $_{k}$ corresponds to the topics in the $k^{\text {th }}$ level on a slide. The level of title is regarded as 0 and itemized level 1 corresponds to 1 .

Here, we show the topic graph which is created by slides in Figure 3(a). Figure 3(b) shows relationships between topics that can be grasped from layout information of the slides. Since the Method slide is located next to the Approach slide, a sequential relationship is attached from the node of Approach to that of Method. Since the Approach slide consists of two topics of the same itemized level, they become child nodes of Approach and sequential relationships are attached between them. On the other hand, the Method slide consists of three topics with two different itemized levels. Therefore, the first topic becomes a child node of Method, and the rest are generated as child nodes of the first topic.

Next, the example of re-organizing relationships is shown. In Figure 3(a), two slides have the words "topic graph" in common. The "topic graph" is introduced in slide 1 and its detailed definition is described in slide 2. Therefore, it is better to arrange the explanation of "topic graph" as a sub-graph of its definition. Let us calculate the importance of "Topic graph: organization of 

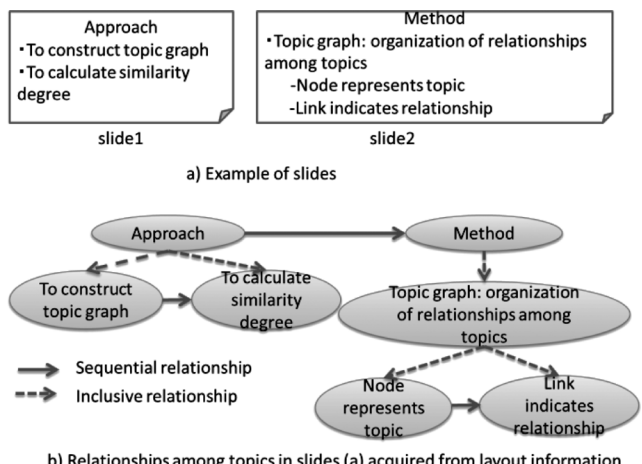

Figure 3. Example of Relationships Acquired from Layout Information.

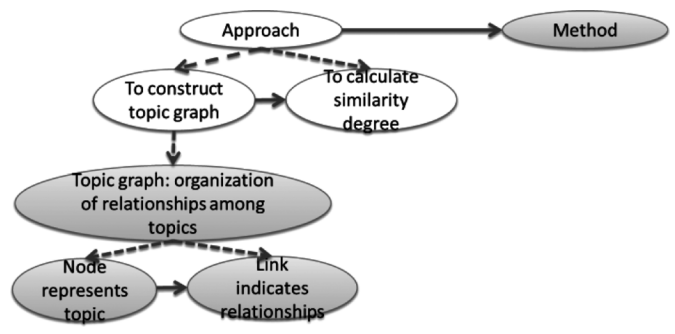

Figure 4. Topic Graph of Slide Figure 3(a).

relationships among topics" in Figure 3(a) as an example. The itemized level of the sentence is 1 . The number of topics in itemized level 1 is 1 and in itemized level 2, it is 2 . Therefore, its importance is calculated as $(1 / 2)^{1 /}$ $\left\{1 *(1 / 2)^{0}+1 *(1 / 2)^{1}+2 *(1 / 2)^{2}\right\}=1 / 4$. If the importance is larger than the threshold, the node is regarded as an explaining topic and its sub-graph is moved as the subgraph of the explained node. If the threshold for the importance is set as $1 / 5$, the node is regarded as an explaining node. Then, the relationships between topics shown in Figure 3(b) are modified and the topic graph shown in Figure 4 is created. In Figure 4, colored nodes are topics of slide 2 .

\section{Detection of Relationships among Slides from Topic Graph}

The relationships between two slides are determined using a topic graph. Usually, slides consist of several nodes in a topic graph. The centered location of all nodes (focus point) on each slide is regarded as the average point of all nodes on the slide. A change in the focus points between slides may express relationships between slides globally. A focus point is represented based on two axes, proceeding degree and depth. The following is the equation for expressing the focus point of slidei:

Focus point $_{i}=\left(\right.$ proceeding degree $_{i}$, depth $\left._{i}\right)$

Nodes that follow the first topic on the first slide by only a sequential relationship (representative topic: $R T$ ) represent the summary of the slides. Proceeding degree indicates the positions of slides in the summary. The following is the equation for determining the proceeding degree of slide $_{i}$ :

Proceeding degree $_{i}=\frac{\sum \text { ID of } R T \times \text { number of nodes in slide } e_{i} \text { in subgraph of } R T}{\text { Number of nodes in slide } e_{i}}$

The ID of RT is the position of RT in the summary from the first RT. The proceeding degree is derived based on the average position in the summary of all nodes on the slide. According to the topic graph shown in Figure 4, the proceeding degree of slide 1 in Figure 3 (a) is calculated as $(1 * 3+2 * 0) / 3=1$, which means that the topic that this slide focuses on is topic 1. Similarly, the proceeding degree of slide 2 is $(1 * 3+2 * 1) / 4=5 / 4$.

Depth corresponds to the level of explanation for each topic. The following is the equation for determining the depth of slide $_{i}$ :

$$
\text { Depth }_{i}=\frac{\sum \text { Layer }_{j} \times \text { Number of nodes in slide } \text { in Layer }_{j}}{\text { Number of nodes in slide }}{ }_{i},
$$

where Layer $_{j}$ corresponds to the number of links to reach the node from RT. Depth is determined by the number of included nodes of slide $i$ for each layer. In the example in Figure 4, the depth of slide 1 is calculated as $(1 * 1+2 * 2) / 3=5 / 3$, while that of slide 2 is $(1 * 1+2 * 0+$ $3 * 1+4 * 2) / 4=3$.

The relationships between slides can be understood by the direction of the vector, whose starting point corresponds to the focus point of slide $_{i}$ and the ending point corresponds to that of slide $_{i+1}$. If two slides have a sequential relationship, the proceeding degree becomes larger and depth may not change. On the other hand, if two slides have an inclusive relationship, depth becomes larger while the proceeding degree may not change. 


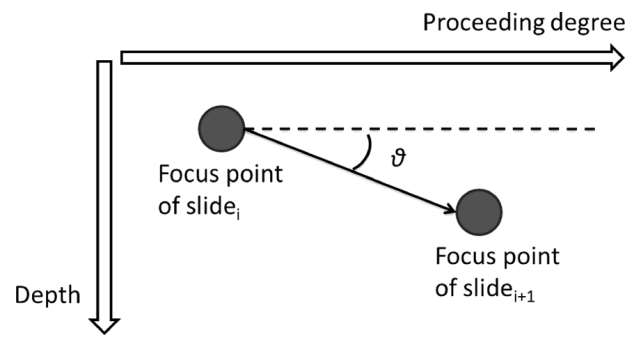

Figure 5. Vector between slide $_{i}$ and slide $e_{i+1}$.

Therefore, if the cosine value of the angle of the vector of $\theta$, as shown in Figure 5, is smaller than the threshold, slides are regarded as having an inclusive relationship. On the contrary, if the cosine value is larger than the threshold $\theta^{\prime}$, slides may have a sequential relationship. Topics with a sequential relationship from one topic and those with an inclusive relationship usually do not have direct relationships, so they are not included in one slide. Thus, two relationships cannot be attached to the same pair of slides. $\theta^{\prime}$ should be smaller than $\theta$, so that the relationships between slides are determined either as a sequential relationship, an inclusive relationship, or no relationship.

Figure 5 is an example of determining the relationship between slides 1 and 2 in Figure 3(a). The focus point of slide 1 is $(1,5 / 3)$ and that of slide 2 is $(5 / 4,3)$, and the cosine value of the vector from slide 1 to slide 2

is $\frac{3}{\sqrt{265}} \doteqdot 0.184$. If the threshold for detecting an

inclusive relationship is set as $0.5\left(\theta=60^{\circ}\right)$, these slides have an inclusive relationship since their cosine value is smaller than the threshold.

\section{Experiment}

We implemented a prototype system, which analyzes the inputted Microsoft Office PowerPoint presentation file and detects the pairs of slides that are in a sequential or inclusive relationship. The following are the steps of the system:

Step 1: analyze layout information and divide the content into sentences;

Step 2: extract keywords from divided sentences and construct a topic graph from them; and

Step 3: determine the pair of slides that have relation-
Table 1. Experimental Results.

\begin{tabular}{|l|c|c|c|c|c|c|c|}
\hline & \multicolumn{3}{|r|}{ Sequential relationship } & \multicolumn{5}{|c|}{ Inclusive relationship } \\
\hline Slides & $2 \rightarrow 4$ & $7 \rightarrow 10$ & $7 \rightarrow 11$ & $4 \rightarrow 6$ & $4 \rightarrow 7$ & $7 \rightarrow 8$ & $15 \rightarrow 16$ \\
\hline A & $\bigcirc$ & $\times$ & $\bigcirc$ & $\bigcirc$ & $\bigcirc$ & $\times$ & $\bigcirc$ \\
\hline B & $\bigcirc$ & $\bigcirc$ & $\bigcirc$ & $\bigcirc$ & $\bigcirc$ & $\bigcirc$ & $\bigcirc$ \\
\hline C & $\bigcirc$ & $\times$ & $\times$ & $\bigcirc$ & $\bigcirc$ & $\times$ & $\bigcirc$ \\
\hline D & $\bigcirc$ & $\bigcirc$ & $\bigcirc$ & $\bigcirc$ & $\bigcirc$ & $\times$ & $\bigcirc$ \\
\hline E & $\bigcirc$ & $\times$ & $\bigcirc$ & $\bigcirc$ & $\bigcirc$ & $\times$ & $\bigcirc$ \\
\hline F & $\bigcirc$ & $\times$ & $\times$ & $\bigcirc$ & $\bigcirc$ & $\bigcirc$ & $\bigcirc$ \\
\hline G & $\bigcirc$ & $\times$ & $\times$ & $\bigcirc$ & $\bigcirc$ & $\bigcirc$ & $\bigcirc$ \\
\hline
\end{tabular}

ships.

C\# is used to implement the entire prototype system and $\mathrm{MeCab}^{(7)}$ is used in Step 2 as a morphological analyzer to divide sentences into words.

We conducted an experiment to evaluate the validity of the estimated slide relationships. Since the objective of this research is to estimate the understanding of a listener, we asked seven undergraduate students ( $A$ to $G$ ) to evaluate each slide relationship estimated by the system. These participants were engaged in research in computer science and had fundamental knowledge of typical presentations in computer science.

The participants were asked to note down their agreement or disagreement for each relationship that the system estimated. Target slides were research presentation slides of a student in our laboratory who was not a participant in the experiment. The slides were created using PowerPoint 2007 and were written in Japanese. There were 16 slides. Slide 2 introduced the background of the research and slide 4 described the objective of the research. Slides 6 and 7 explained the approach and slides 8 to 13 were the details of the mechanisms. Slides 14 to 15 showed the experiment and its results, and slide 16 was the conclusion of the presentation. Some slides originally contained diagrams, but they also had text indicating the content of the diagrams. When extracting the relationship, diagrams were eliminated from slides. Participants also evaluated slides without diagrams.

The system extracted three sequential relationships and four inclusive relationships. Table 1 lists the results. In the table, " $2 \rightarrow 4$ " indicates the relationship between slides 2 and $4, \bigcirc$ means that the participants agreed with the relationships the system extracted, and $\times$ 
means they disagreed. For two sequential relationships and three inclusive relationships, more than half of the participants agreed with the system's detection.

The relationships extracted correctly were those between background and objective, objective and approach, and experimental results and conclusion. These topics were described using only text and are typical in research presentations in computer science. Therefore, this author knew reasonably well how to create effective slides that reflect the topic flow.

The relationships that were selected as inappropriate by more than one participant were those between approach and the detailed explanation of the methods. The way of introducing the method depends on the content of each study, and there is no typical style for describing the topics.

The title of slide 8 was "data structure of the text". On slide 7, the keyword "text" was included, so the system assigned inclusive relationships between slides 7 and 8 . However, many participants regarded "data structure of the text" and "text" as different keywords because the former is a compound noun. They thought that slide 8 did not explain slide 7 in detail, but it presents the next topic. Currently, our system treats a compound noun as two different nouns because of the output of $\mathrm{MeCab}$. As the experimental results showed, impressions for the compound noun differed among participants. Therefore, as future work we need to investigate the intention of various audiences regarding various compound nouns and we need to consider the way of treating a compound noun.

On the other hand, most participants thought there were no relationship between slides 7 and 10 and slides 7 and 11 since there were only a few common words. Currently, a sequential relationship between topics is attached based on the layout information and order of slides. However, in some slides, it is difficult to find any relationship even if they are next to each other. Thus, the method of determining a sequential relationship should be modified, e.g. to address the number of common keywords.

\section{Conclusion}

We proposed a presentation slide creation support system which automatically detects relationships between slides that can be estimated from the layout and lexical information on the slides. Based on the results, our system could correctly detect relationships if slides consisted of typical topics of a presentation and did not contain compound nouns. However, the relationships between slides that contain compound nouns were differently understood by audience members. We need to devise a method for handling compound nouns.

This research was only focused on slides that mainly consisted of text. However, typical slides often contain diagrams and tables. Estimating topics and their relationships from diagrams is more difficult than from text, since ways of drawing diagrams differ among authors even for the same content. Therefore, specific knowledge on reading diagrams should be developed and included in the system in order to analyze topics from diagrams.

Furthermore, in some presentations, slides are used as supplementary material to the author's speech. To evaluate the created slide in such a presentation, not only content on the slides but also that of the speech are important. The current estimation method should be extended to the speech text in order to support creation of such slides.

The objective of our research was to show the differences between slides that can be understood by the audience and the intention of the author. Currently, our system does not address how to estimate the author's intention. It may be a burden for authors to indicate the relationships between all pairs of slides beforehand. Also, some authors do not take into account relationships when creating slides. On the other hand, there is a typical presentation flow for each presentation genre. For example, research presentations in computer science start from the background followed by the objective. Then, the approach is explained and detailed mechanisms are introduced. After the usability of the mechanism is discussed according to the experimental results, the presentation is concluded. Relationships of such typical topics for each presentation theme can be easily defined. Therefore, we believe that asking authors to select typical topics corresponding to each slide is the easiest way of estimating an author's intention.

We should also devise a method for indicating differences between slides and intentions of authors. Authors need to know the pair of slides at which the audience cannot follow the story. In addition, they need to understand the reason why the story cannot be followed. To directly give the reason, however, prevents authors from considering such issues by themselves. Therefore, 
a mechanism for generating messages that promote meta-learning of slide creation should be developed.

\section{References}

(1) Okamoto, R. and Kashihara, A.: "Back-Review Support Method for Presentation Rehearsal Support System", in Proc. of KES 2011, Part II, LNAI 6882, ed. König, A. et al., pp. 165-175, Springer, Heidelberg (2011).

(2) Kashihara, A., Saito, K. and Hasegawa, S.: "A Cognitive Apprenticeship Framework for Developing Presentation Skill”, IEICE Technical Report, Vol. 111, No. 141, pp. 23-28 (2011) (in Japanese).

(3) Maeda, K., Hayashi, Y., Kojiri, T., and Watanabe, T.: "Skill-up Support for Slide Composition through Discussion”, in Proc. of KES 2011, Part II, LNAI 6883, pp. 637-646, Springer (2011).

(4) Kamewada, K. and Nishimoto, K.: "Supporting Composition of a Presentation by Showing Transitions of Audiences' Attentions", Trans. of Information Processing Society of Japan, Vol. 48, No. 12, pp. 3859-3872 (2007) (in Japanese).

(5) Hanaue, K. and Watanabe, T.: "Externalization Support of Key Phrase Channel in Presentation Preparation", International J. of Intelligent Decision Technologies, Vol. 3, No. 2, pp. 85-92 (2009).

(6) Tufte, E. R.: The cognitive style of PowerPoint: pitching out corrupts within, Graphic Press, Cheshire, CT (2006).

(7) Salton, G., Fox, E. A. and Wu, H.: "Term-weighting Approaches in Automatic Text Retrieval, J. of Information Processing \& Management, Vol. 24, No. 5, pp. 513-523 (1988).

(8) MeCab: Yet Another Part-of-Speech and Morphological Analyzer, http://mecab. sourceforge.net/ (2009).

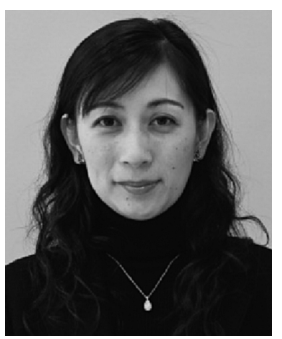

Tomoko Kojiri received the B.E., M.E., and Ph.D. degrees from Nagoya University, Nagoya, Japan, in 1998, 2000, and 2003, respectively. From 2003 to 2007, she was a research associate at Nagoya University. From 2007 to 2011, she was an assistant professor in Nagoya University. Since 2011, she has been an associate professor in the Faculty of Engineering Science, Kansai University, Japan. Her research focus is on developing information systems which support intelligent human activities. Her research interests include intelligent tutoring systems, computer-supported collaborative learning, human-computer interaction, meta-learning support, presentation support, etc. She is a member of IPSJ, JSAI, IEICE, JSET, JSiSE, APSCE, and KES International.

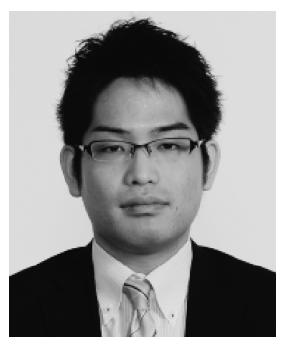

Fumihiro Yamazoe received the B.E. degree from Kansai University, Japan, in 2011. His research interests include creativity support systems. 